\title{
Rearrangements in three-body decaying resonances
}

\author{
AS Jensen ${ }^{1}$, DV Fedorov ${ }^{2}$ and E Garrido ${ }^{3}$ \\ ${ }^{1,2}$ Department of Physics and Astronomy, Aarhus University, DK-8000 Aarhus C, \\ Denmark \\ ${ }^{3}$ Instituto de Estructura de la Materia, CSIC, Serrano 123, E-28006 Madrid, Spain \\ E-mail: ${ }^{1}$ asj@phys.au.dk, ${ }^{2}$ fedorov@phys .au.dk, ${ }^{3}$ e.garrido@iem.cfmac.csic.es
}

\begin{abstract}
Three-body decays of many-body nuclear resonances are processes where $N$ particles in a quasi-stable configuration is divided in three fragments. The momentum distributions of the fragments carry the information of the resonance and the decay process. Calculated results should be compared to accurate and complete measurements with the purpose of extracting such information. Two almost independent problems must be solved before fully reliable results become available. First contraction of the $\mathrm{N}$-body degrees of freedom to those of three particles has to be consistently achieved. This presents a conceptual problem since it implies matching of rather incompatible models and the related effective interactions. The second problem is that the resonance structure often furthermore undergoes major changes from small to large distances. The couplings causing these changes may in principle be known within a given three-body model, but even under this assumption, the accuracy requirements are in difficult cases very hard to meet. Different reasons apply to different cases. One example is when very small couplings extend over large distances as for prominent substructures. We illustrate these two open problems with a number of nuclear three-body decays. We emphasize that these problems are the simplest of a much more advanced series of multi-body decays and reaction processes proceeding from $N$ particles to three-body clusters.
\end{abstract}

PACS numbers: 21.45.-v, 21.60.Gx, 24.30.Gd 


\section{Introduction}

The nuclear N-body system has in general a number of bound and excited states, some of which decay by emission of clusters of nucleons. This immediately implies that description of such decays involves continuum structures. Since all bound nuclear clusters except for the neutron are charged the long-range Coulomb interaction is almost always present. Obviously binding is provided by the short-range strong interaction. Thus in decays we are in general dealing with $N$-body degrees of freedom contracting into much fewer cluster degrees of freedom. The process obeys the laws of quantum mechanics, which, to determine the decisive continuum structures, require simultaneous treatment of both small and large distances for combinations of short and long-range interactions

The structure problem is in general already extremely difficult as evidenced by the fact that ab initio computations of bound nuclear $N$-body states at the moment only are within reach when $N$ is smaller than 12. Descriptions of decaying structures into few fragments add to the difficulties due to the complications with reduction of degrees of freedom, and simultaneously contributing short and long-range interactions. Two examples of two-body decaying systems, $\alpha$-emission [1] and binary fission [2], suffice to illustrate the difficulties.

In the early days of quantum mechanics $\alpha$-emission was an unavoidable phenomenon that convinced physicists of the necessity to embrace the new theory. The tunneling probability through centrifugal and Coulomb barrier from nuclear surface to infinity accounted for variations in the $\alpha$-decay rate by 30 orders of magnitude [3]. Energy and momentum conservation determines the energy of the $\alpha$-particle and the daughter nucleus. The problem of contracting the degrees of freedom enters only when details of the decaying short-distance structure are important. This is taken care of by phenomenological spectroscopic or preformation factors [3] which are necessary to account for the remaining discrepancies of one to two orders of magnitude.

Binary fission is another complicated two-body decaying structure [4]. Now the short-distance structure is all decisive for which fragments emerge after the divison in two pieces. Obviously $\alpha$-emission can be viewed as an extremely asymmetric fission. The mass distribution of the fission fragments emerges after the process of contracting the many degrees of freedom down to two is completed. The decay rate is in contrast to $\alpha$-decay far from determined by tunneling through Coulomb and centrifugal barriers. This process has to be described by a complicated combination of diffusion, evaporation and dynamic evolution [5].

The simplest extension is from two to three-body decaying structures [6]. All the problems of two-body decays remain and in addition now the energy can be distributed continuously between the three particles without violating energy and momentum conservation. The unstable beam facilities combined with detector development have recently allowed accurate and kinematically complete determination of the fragments arising from three-body decays, see e.g. [7]. These distributions are far from understood. 
Several questions are still to be fully answered: How to perform model calculations and compare in details to measurements [8]?, and even more challenging how much can model-independently be inferred from the measured large-distance to the initially decaying short-distance structures?

We shall in this report formulate and discuss the problems illustrated by the decay process of a resonance. We shall assume that this continuum structure is populated in beta-decay or by an unspecified reaction. In this way we simplify the story by omitting everything prior to population of the resonance. This excludes discussions of some reactions but allows focus on decay mechanisms of resonances and analysis of possible model independent connections to measurements. This seems to be a reasonable beginning since resonances are cornerstones in continuum structures. In any case the idea is that detailed formulation of a problem often helps to solve it.

\section{Formulation}

We assume a resonance is formed by a system of $N$ particles. We imagine population occurred by a reaction or through beta-decay. However, the formation history does not influence the subsequent decay. Before proceeding we have to define a resonance. Our choice is usually the wavefunction corresponding to the complex energy of a pole in the $S$-matrix or equivalently the wavefunction with a complex energy and only outgoing flux in all channels. It is also possible to piece a resonance together by continuum wavefunctions with real energies in an appropriate interval where the cross sections are strongly varying and substantially above the background.

Let us focus on three-body decays and three-body computations even though most of the following applies more generally or easily can be extended. We use our resonance definition which implies some analytical extension into the complex plane which can be achieved by complex scaling of the coordinates [9]. The advantage is that the resonance wavefunction is a solution to the (complex scaled) ordinary Schrödinger equation with bound-state boundary conditions. The disadvantage is that the complex scaling method only provides solutions for relatively narrow resonances.

The overlap of the resonance wavefunction with asymptotic large-distance outgoing plane waves provides the momentum distributions of the emerging three particles [10]. This is loosely speaking the Fourier transform at large distance of the resonance wavefunction. The main difficulty of diverging Fourier integrals is solved by the Zeldovic regularization. The momentum distributions are determined by the momentum-space wavefunction multiplied by a Breit-Wigner shape in three-body resonance energy and width.

The procedure can in the simplest version be formulated as computing a threebody resonance by complex scaling, Fourier transforming by Zeldovic regularization, and integrating over all momenta except the observables of interest. The simplifying assumptions are three-body structures and narrow resonances defined by complex scaling. This allows a relatively clean presentation of the problem. 


\section{Open problems}

Decay of a many-body resonance into three fragments presents two problems. The first also appears in two-body decay, i.e. reduction of the $N$-body degrees of freedom relevant at small distance to those describing the (two or) three fragments at large distance where the decay products emerge. The second problem arises when the three-body resonance wavefunction is allowed to change character from small to large distance. How to reduce the degrees of freedom, and how to understand and incorporate properly the couplings causing a dynamical evolution.
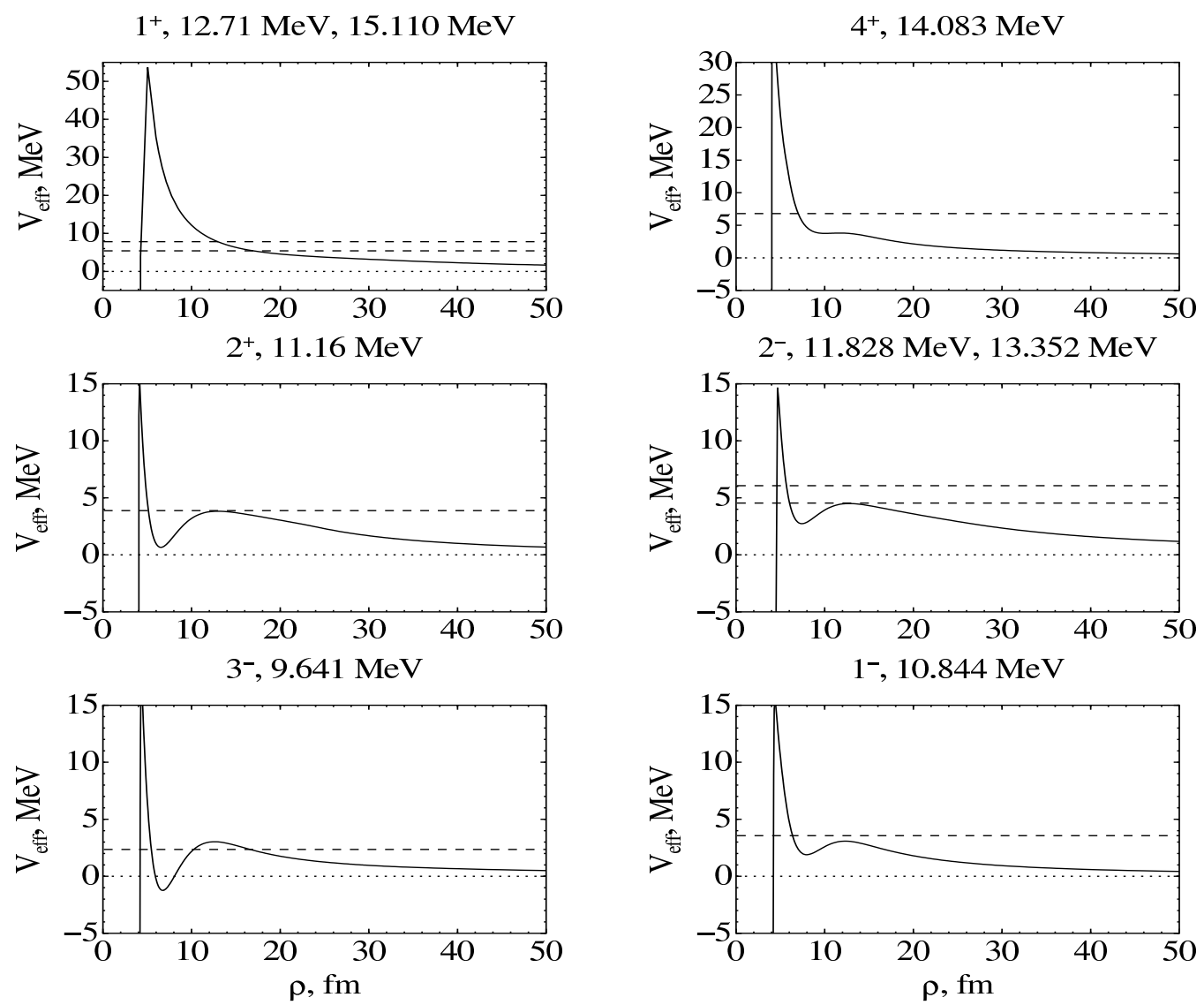

Figure 1. The computed lowest adiabatic potential as function of hyperradius for a number of resonances of ${ }^{12} \mathrm{C}(\alpha+\alpha+\alpha)$. The horizontal lines mark the resonance energies measured above the three-body threshold. The corresponding excitation energies are given above each of the panels.

\subsection{Contraction of superfluous degrees of freedom}

The decay into three particles prescribes use of a three-body model at the (large) distances where the fragments have gained their final identity. On the other hand, at small distances all $N$ particles interact and the final state cluster structures are sometimes far from being realized. Numerous examples exist, e.g. nuclear resonances 
with a small partial width for decay into three fragments, where the small width can be attributed to a total lack of cluster structure at small distances, see f.ex. the structure and decay of $1^{ \pm}$states in ${ }^{12} \mathrm{C}$, see $[11,12]$. Thus the many-body structure should be smoothly joined to the three-body cluster structure.

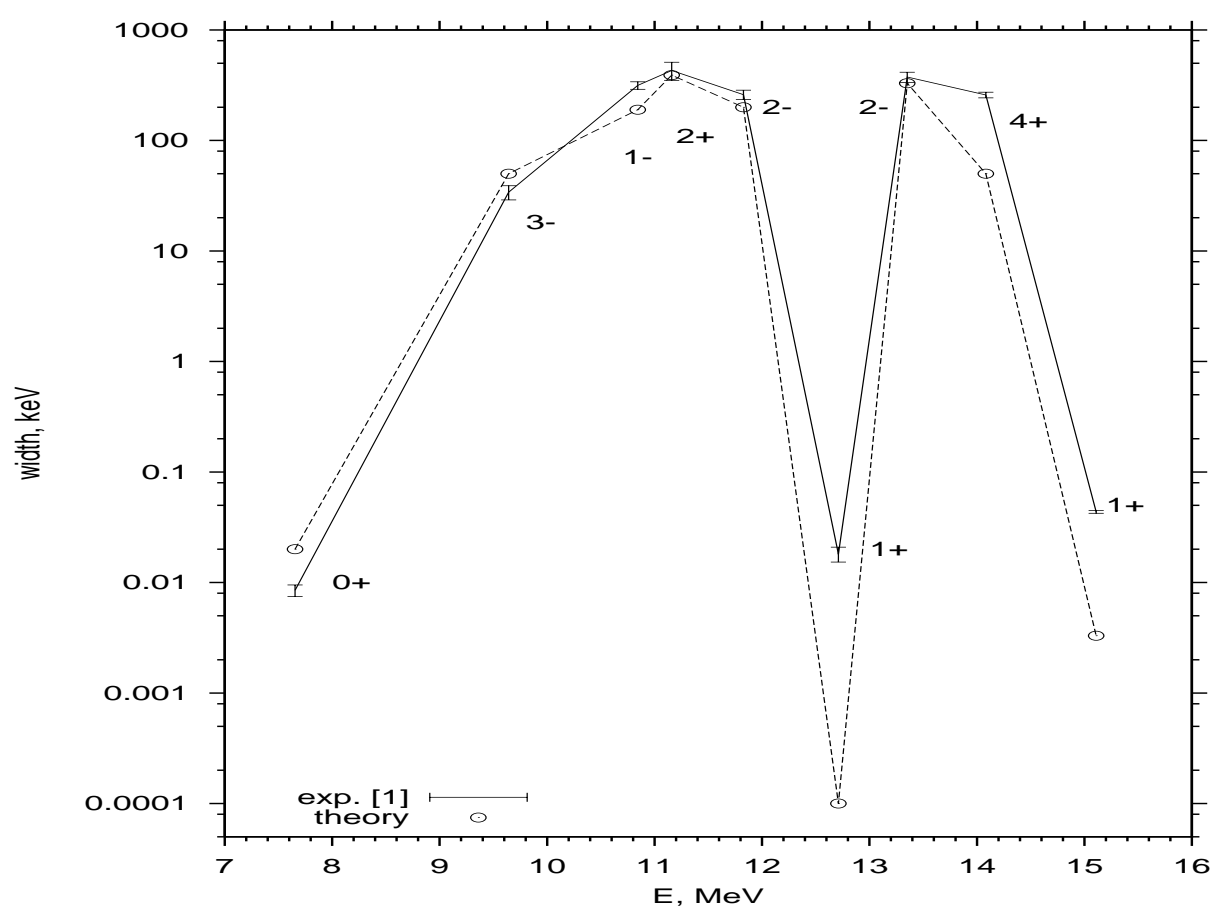

Figure 2. The computed widths compared to the measured values for different resonances of ${ }^{12} \mathrm{C}$ with excitation energy $E$. The WKB approximation is used for tunneling through the lowest adiabatic potential where the inner turning point is fixed to a hyperradius of $\rho=4 \mathrm{fm}$.

One could try two extremes of either full $N$-body or only three-body models for all distances. The latter case is the usual three-body problem for point-like particles where boundary conditions both at zero and infinity are used. Then the short-distance behavior is crucial for occurrence and properties of resonances. It is from the beginning unclear from which distance it is sufficient to use a description in terms of cluster structure.

One strong indication is from tunneling through the barrier of the dominating adiabatic three-body effective potentials. We illustrate the idea in Fig. 1 by the ${ }^{12} \mathrm{C}$ resonances [13]. In some cases a minimum is found in the three-body computation but far from always. This means that the short-distance structures of the resonances are far from the cluster structure emerging after the decay, because a minimum inside a barrier is needed to produce a resonance.

If we artificially introduce a strong square-well attraction at short distance the resonance energy can be adjusted to the meassured position and tunneling through the remaining barrier determines the liftetime. In Fig.2 we show the WKB estimates of these lifetimes. They vary by five orders of magnitude but are within one order 
of magnitude correlated with the tunneling probability. This result is not trivial as the energy and angular momentum dependence is completely different from those of the well-known two-body decay modes. The conclusion seems to be that the cluster structure is established at rather small distances roughly corresponding to a common nuclear surface. However, closer inspection reveals that the states traditionally denoted as "shell model" states, in contrast to cluster states, are further away from three-body lifetime estimates. In any case to get accuracies within an order of magnitude requires more short-distance details than provided for point-like cluster particles.

It then seems obvious to perform $N$-body shell-model computations which are designed to account for short-distance correlations, and known to have severe difficulties in describing large-distance and cluster properties, see e.g. [14]. Then combine these shell-model results with three-body computations at small to intermediate distances corresponding to about nuclear surface radii. This procedure seems to be straightforward. However, in practice it has not been consistently implemented due to conceptual, as well as technical, problems. Assume that the results of a large shell model computation is expanded in a basis of three-body relative wavefunctions multiplied by intrinsic cluster wavefunctions. Under the very uncertain assumption that shell-model accuracy is sufficient up to distances where the cluster structures have separated out, we have in principle established new boundary conditions for the three-body computation. At best we have then a six-dimensional surface in relative three-body coordinates but supplemented by the problem related to the center-of-masses of total and individual clusters. The shell-model energies are far from the high accuracy obtained in the threebody calculations. Therefore they cannot be used as guides. Still all clusters may not be established at the same time, one could come first whereas the other two only appears after the first has moves away. The Pauli principle has to be obeyed for identical particles inside and across individual clusters. The change from $N$ to three particles requires a change of interactions, and furthermore a change of Hilbert space which in turn requires a change to correponding effective interactions [15]. Since the effective cluster-cluster interaction cannot be derived from the nucleon-nucleon interaction, this presents a conceptual problem.

It would help to employ a similar basis in the $N$ and three-body computations but that is also very difficult since the three-body continuum is necessary but this is easily in conflict with completeness of the shell-model basis. Thus it is an open problem to contract the degrees of freedom from $N$ to three particles.

\subsection{Dynamical evolution of the three-body structure}

Within the three-body model with point-like particles a crucial change of structure may occur from small to large distance. Small distances are decisive for the resonance properties and supply the largest part of the energy, but large distances reflect the fragment distributions after decay. These structures are linked continuously by quantum mechanics. The change of structure may occur for a variety of reasons. The cluster 


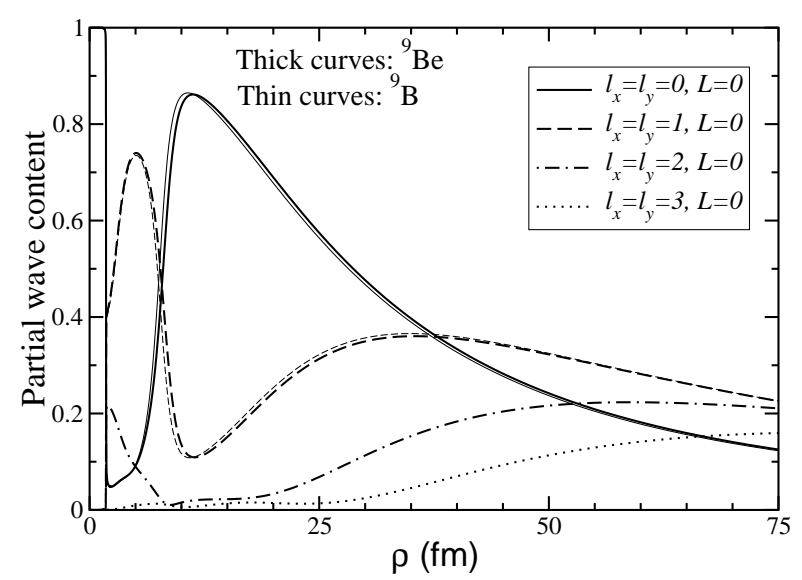

Figure 3. The partial wave decomposition of the dominating adiabatic angular wavefunction for ${ }^{9} \mathrm{Be}$ and ${ }^{9} \mathrm{~B}$ as function of hyperradius $\rho$. The partial angular momenta $l_{x}$ and $l_{y}$ refer to the $\alpha$-nucleon and the $\alpha$-( $\alpha$-nucleon) Jacobi coordinates.

structure of ${ }^{9} \mathrm{Be}\left({ }^{9} \mathrm{~B}\right)$ consists of two $\alpha$ 's and a neutron (proton). At small distance the $\alpha$-nucleon $p_{3 / 2}$-interaction provides the attraction crudely speaking with the nucleon between the two $\alpha$-particles. At large distance $s$-waves are preferred and ${ }^{8} \mathrm{Be}$ in the (unbound) ground state moves away from the neutron. The dramatic change of partial wave decomposition with distance is shown in Fig.3. The dominating $p$-waves $\left(l_{x}=1\right)$ below $\rho=7.5 \mathrm{fm}$ are quickly replaced by $s$-waves $\left(l_{x}=0\right)$ for larger hyperradii.

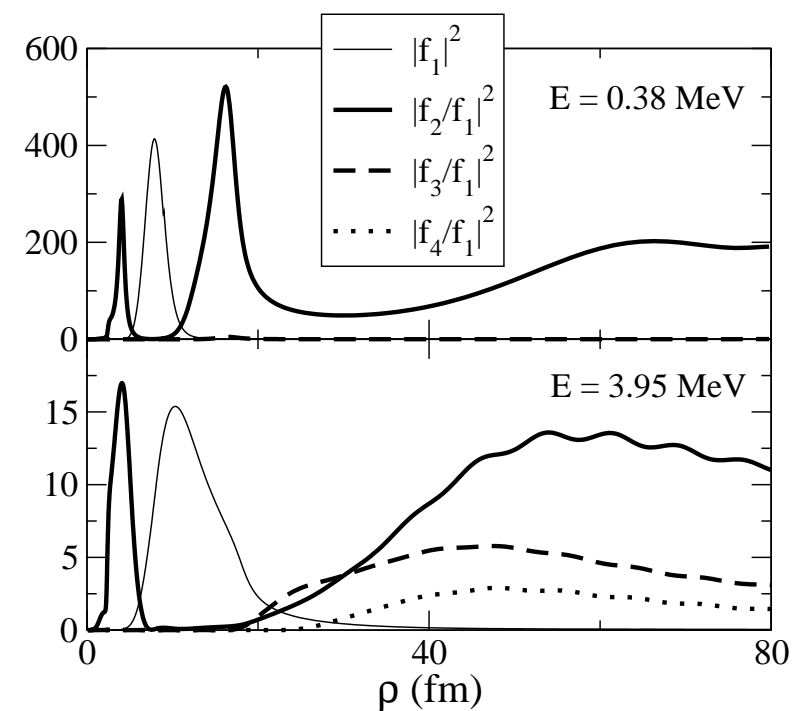

Figure 4. The ratio of probabilities for the four lowest adiababtic potentials as functions of hyperradius, $\rho$, for each of the two $0^{+}$resonances of ${ }^{12} \mathrm{C}$ at $0.38 \mathrm{MeV}$ and $3.95 \mathrm{MeV}$ above threshold or at excitation energies of $7.63 \mathrm{MeV}$ and $11.2 \mathrm{MeV}$. The ratios to the dominating probability $\left|f_{1}\right|^{2}$ are given for both states. 
In general, the lowest energy is favored in adiabatic evolution but if possible the system may prefer to maintain the structure in diabatic evolution [16]. In actual cases a fraction of each can be expected as the resulting compromise between lowest energy or structure change [17]. The amounts of each can vary from 0 to $100 \%$ are detemined by the couplings. This can be seen for the two lowest $0^{+}$resonances of ${ }^{12} \mathrm{C}$ as shown in Fig.4. The probablilities vary strongly for occupying each of the adiabatic potentials. The Hoyle state at $0.38 \mathrm{MeV}$ consists by far mostly of the lowest component for all distances. In contrast, the next $0^{+}$resonance at $3.95 \mathrm{MeV}$ is at large distance mostly of the same structure as the Hoyle state but leaving about $10 \%$ of a different component at large distance.

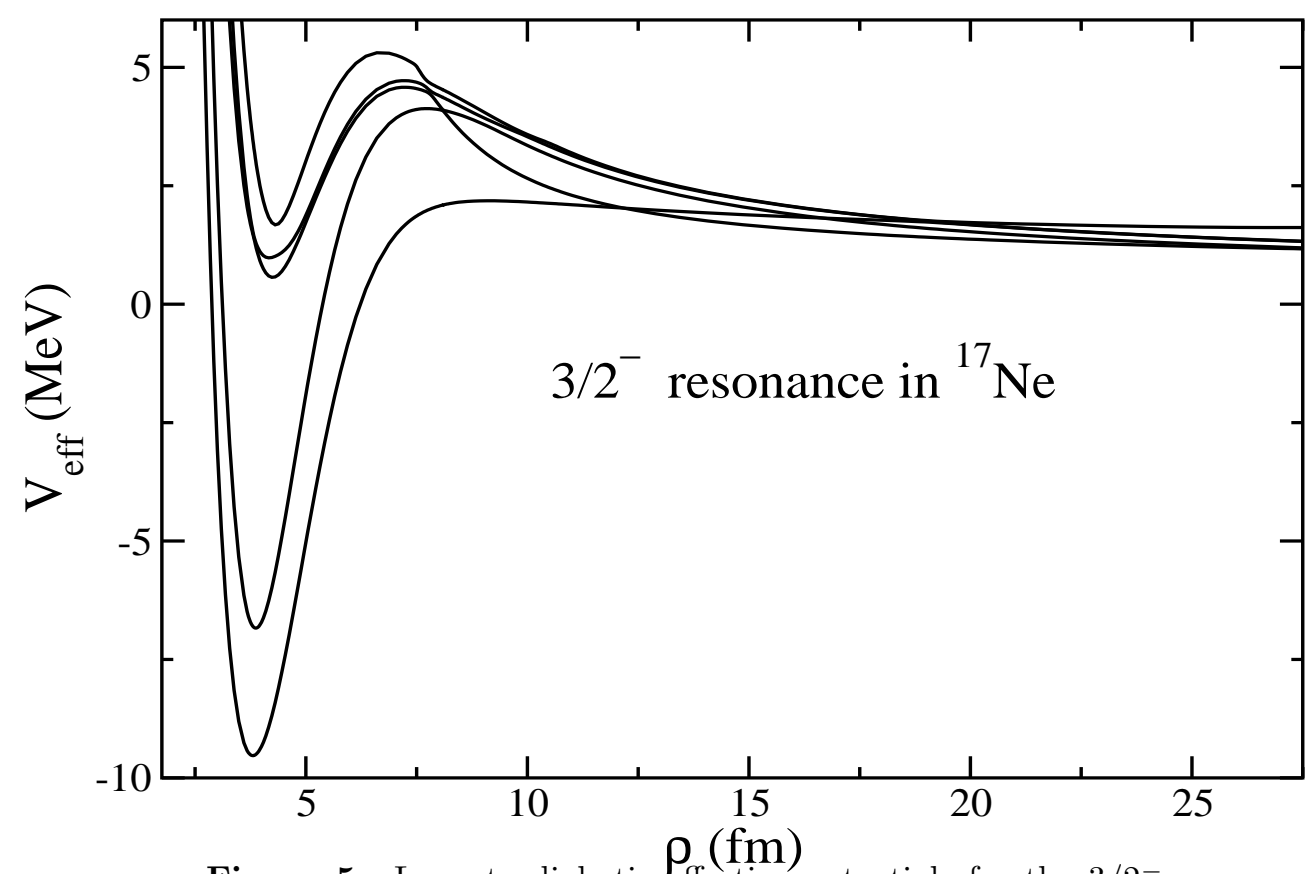

Figure 5. Lowest adiabatic effective potentials for the $3 / 2^{-}$resonance in ${ }^{17} \mathrm{Ne}$ at $0.34 \mathrm{MeV}$. The interactions are described in [18]. The deepest potential (black curve) describes the ${ }^{16} \mathrm{~F} d$-wave resonance and the last proton in an $s$-wave.

There may be more than one type of these coupled structures and corresponding attempts to change characteristics as e.g. from symmetry due to identical particles [8]. It could also be that only a virtual change occurs if energy conservation forbids the process [18]. For example, a low-lying excited state of one of the clusters may give an important contribution to the resonance structure at relatively small distance. This channel may still be closed in the resonance decay and rearrangements are necessary. These decays can not occur as purely sequential via a lower-lying resonance in a subsystem. This is the decay analog of Borromean structure. All subsystems have no resonance in the energy interval from zero to the decaying resonance energy scaled by the mass ratios determined by momentum conservation. The energy is measured relative to the energy where all particles are far apart.

This Borromean decay happens for the first excited state, the $3 / 2^{-}$resonance in the 
Borromean nucleus ${ }^{17} \mathrm{Ne}$, which only can decay into two protons and the ${ }^{15} \mathrm{O}$ core. We show in Fig.5 the adiabatic potentials where the deepest, after the barrier, decreases very slowly. The structure corresponds to the $d$-wave resonance of ${ }^{16} \mathrm{~F}$ with the last proton in an $s$-wave, and the (almost) antisymmetric configuration of ${ }^{16} \mathrm{~F}$ in an $s$-wave with the last proton in a $d$-wave. The decrease matches the Coulomb and centrifugal potentials of one proton relative to the spatially more confined two-body configurations of ${ }^{16} \mathrm{~F}$. The ${ }^{17} \mathrm{Ne}$ resonance is dominated by such a structure, even after other potentials have crossed and appear with lower energies. This is then an example of maintaning the structure rather than following the lowest energy. However, this can not continue to infinity because the energy is too small. The ${ }^{16} \mathrm{~F}$ structure must exchange energy with the last proton in order to conserve the total energy in the decay process. Thus, the decay mechanism is virtual sequential decay where the energetically forbidden ${ }^{16} \mathrm{~F}$ structures are exploited as vehicles until the last proton is at the edge of the barrier.

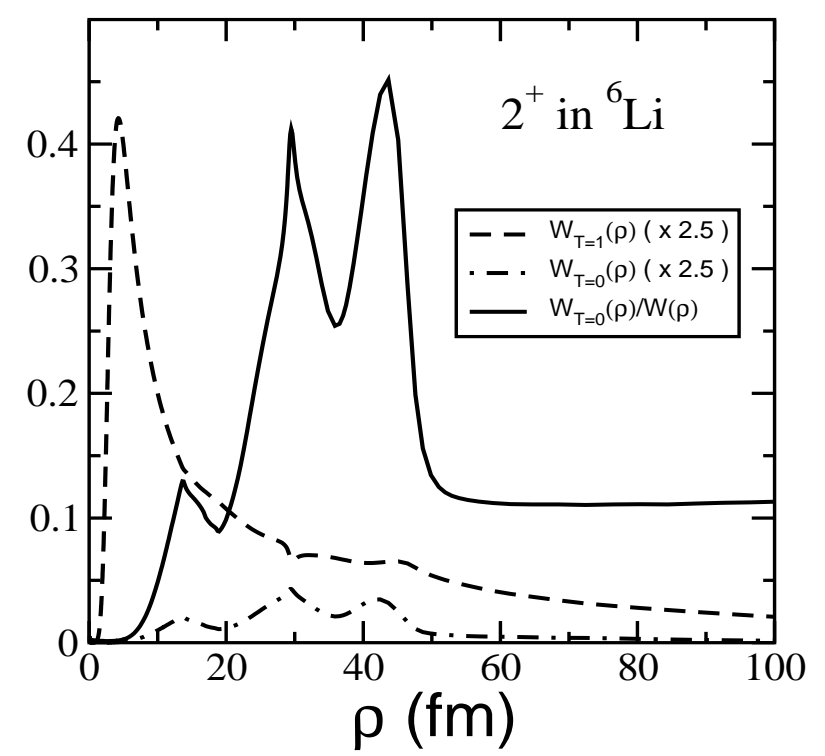

Figure 6. The probability of finding isospin 1 and 0 as function of hyperradius $(\rho)$ for the $2^{+}$state in ${ }^{6} \mathrm{Li}$.

An important example is symmetries arising from quantum statistics and nearly fulfilled conservation laws [8]. Small violation as for the weakly broken isospin causes a very weak coupling between states related to adiabatic potentials of different isospins [17]. Such states would mix but the accuracy requirement might be overwhelmingly demanding. Imagine population of an almost isospin pure $T=1$ resonance in ${ }^{6} \mathrm{Li}$ which decays by neutron plus proton emission. The remaining $\alpha$-particle has isospin zero but the isospin 1 neutron-proton system couples weakly to the isospin 0 deuteron with the much lower energy. The coupling is caused by the Coulomb interaction and efficient where it dominates, i.e. outside the range of the strong interaction, which in turn means at intermediate and large distances where the accuracy is especially difficult to maintain. This is illustrated in Fig. 6 where the abrupt change from isospin 1 to 0 
at $\rho \sim 20 \mathrm{fm}$ is followed by an equally abrupt change at $\rho=48 \mathrm{fm}$ to a steady level at about $10 \%$ for large $\rho$.

The violation causes the system to change structure at intermediate distances and fall down in energy to a lower-lying state of different sysmmetry. Three- $\alpha$ decays of isospin 1 states in ${ }^{12} \mathrm{C}$ must change structure before the three $\alpha$-particles are formed. This means at small distances where the many-body structure is dominant. This is reflected in the potential of $1^{+}$state in ${ }^{12} \mathrm{C}$, see Fig.1.

Another general scenario, a two-body resonance can keep two of the particles close together while the third is moving far away before this resonance decays. This is sequential or virtual sequential two-body decays which can be dealt with separately [19], see Fig.3 and Fig.5, respectively. However, if the lifetimes of the initial threebody system and the two-body resonance are comparable this sequential decoupling cannot be made. Then one cluster is formed and emitted (slightly) before the other two clusters emerge from the $N$ particles. The boundary conditions in the contraction from $N$ particles to 3 clusters is now ill-defined since the (small) distance is ill-defined, see previous subsection. When a two-body substructure is prominent and present at intermediate distances, it has to decay or couple to the three-body continuum.

Then the pertinent questions are if the couplings responsible for the structure rearrangments are defined, known, and possible to treat in practice. Obviously couplings to neglected degrees of freedom are absent and most likely related to the problem of contracting the degrees of freedom. However, the resulting wavefunctions in combination with the correspondingly renormalized operators contain all the information. The couplings within the remaining (three-body) Hilbert space are in principle described in the (three-body) model from the initial two-body interactions. However, these (twobody) interactions are as hard to get as the renormalization due to reduction of Hilbert space. Phenomenology is then almost inevitable, but spatial shape is unspecified and the accuracy requirements can be very severe. Further complications are possible since the influence of couplings can appear anywhere from small to large distances.

In particular, it is difficult when more than one substructure coherently contribute and couple perhaps at different distances. It helps to employ Faddeev equations which allow simultaneous treatment of different subsystems on the same footing. For longlived substructures the couplings are tiny and continuously occurring until the two-body decay has taken place or equivalently until no trace remains of the two-body structure. The Coulomb interaction enhances these difficulties as the Coulomb coupling extends to infinity. Combinations with Efimov-like resonance structures of extreme spatial extensions probably cannot be dealt with numerically [20]. Incompatibility between analytic derivations, phenomenological and numerical couplings can be disastrous.

In total, the rearrangements within the possible three-body structures are in principle determined by well defined couplings. Unfortunately, the technical problems are tremendous in many cases although possible to handle with a fair accuracy in other cases. In close connection it is necessary to address the question of how detailed information about the fragment distributions can be used to deduce decay mechanism 
and initial structure or extract as severe constraint as possible. These are open problems.

\section{Summary, Conclusions and Perspectives}

We have presented the open problems related to understanding of three-body decay processes of $N$-body resonances. The key issues are first that the initial degrees of freedom must be reduced to describe the final state of three clusters, and second that even three-body resonance structures "dynamically" evolve from small to large distances. The first issue involves a many-body structure which has to be smoothly joined to a three-body structure. The second issue is a few-body problem but with the interaction input either fully phenomenologically or derived from properties of the same $N$-body system as for the first issue.

The thresholds for breakup into different parts play a crucial role. When the nucleon driplines are approached these thresholds tend to approach each other and wash out the differences between dynamical evolution and contraction of degrees of freedom. The reason is that reduction could be in steps as already indicated by three-cluster formation via two subsequent two-cluster processes. In cold atomic gases the two-body interaction can effectively be changed. The closest we get to that in nuclei is by electron screening of the Coulomb repulsion. The thresholds for combination of two and three $\alpha$-particles into ground state of ${ }^{8} \mathrm{Be}$ and first $0^{+}$resonance of ${ }^{12} \mathrm{C}$ then both approach zero [21].

We have in this paper indicated how to proceed in special cases but we focussed here more on the problems than on the previous attempts. The formulated problem is only a small piece, perhaps the simplest, of the general issue of decays of $N$-body resonances, e.g. into more than three pieces. It is also only a small piece of reaction descriptions where a resonance is not an intermediate stepping stone, e.g. for short-lived resonances where the lifetime is comparable to the reaction time or when resonance structures are circumvented all-together.

Treating broad resonances introduces a number of other problems than discussed here. It first of all raises questions about a proper definition of resonances as a quasistationary state. If the total energy is off the center of the resonance, the decay is asymmetric as higher and smaller energies "see" smaller and larger barriers, respectively. This suggests that broad resonances are better treated by staying on the real axis while varying the energy. However, this essentially implies that the resonance concept is not used and at the same time knowledge of the population history is required, and even this simplification disappears.

Acknowledgment. Useful comments from K. Riisager are highly appreciated.

\section{References}

[1] Gamow G 1928 Zeit. f. Phys. 51204

[2] Bohr N and Wheeler JA 1939 Phys. Rev. 56426

[3] Siemens PJ and Jensen AS 1987 Elements of nuclei. Many-body physics with the strong interaction (Lecture notes and supplements in physics, Addison-Wesley) 
[4] Brack M, Damgaard J, Jensen AS, Pauli HC, Strutinsky VM, and Wong CY 1972 Rev. Mod. Phys. 44320

[5] Hofmann Helmut 2008 The Physics of Warm Nuclei with Analogies to Mesoscopic Systems (Oxford Studies in Nuclear Physics, Oxford University Press)

[6] Garrido E, Fedorov DV, Fynbo HOU and Jensen AS 2007 Nucl. Phys. A 781387

[7] Blank B et al. 2003 C.R. Physique 4521

[8] Fynbo HOU, Álvarez-Rodríguez R, Jensen AS, Kirsebom OS, Fedorov DV, Garrido E 2009 Phys. Rev. C 79054009

[9] Fedorov DV, Garrido E, and Jensen AS 2003 Few-body systems, 33153

[10] Garrido E, Fedorov DV, Fynbo HOU and Jensen AS 2007 Nucl. Phys. A 781387

[11] Álvarez-Rodríguez R, Garrido E, Jensen AS, Fedorov DV, and Fynbo HOU 2007 Eur. Phys. J. A 31303

[12] Álvarez-Rodríguez R, Jensen AS, Garrido E, Fedorov DV, Fynbo HOU 2008 Phys. Rev. C77 064305

[13] Fedorov DV, Jensen AS and Fynbo HOU 2003 Nucl. Phys. A 718 685-687c

[14] Forssen C, Caurier E, Navratil P 2009 Phys. Rev. C79 021303

[15] Christensson J, Forssen C, Åberg S, and Reimann SM 2009 Phys. Rev. A79 012707

[16] Garrido E, Fedorov DV, Jensen AS and Fynbo HOU 2005 Nucl. Phys. A 76674

[17] Garrido E, Fedorov DV, Fynbo HOU and Jensen AS 2007 Phys. Lett. B 648274

[18] Garrido E, Fedorov DV and Jensen AS 2004 Nucl. Phys. A 73385

[19] Álvarez-Rodríguez R, Fynbo HOU, Jensen AS, Garrido E 2008 Phys. Rev. Lett. 100192501

[20] Garrido E, Fedorov DV and Jensen AS 2006 Phys. Rev. Lett. 96112501

[21] Jensen AS, Fedorov DV, Langanke K, and Müller H-M 1995 Three alpha-particle system in a dense helium plasma (ENAM 95, Int. Conf. on exotic nuclei and atomic masses, eds. Simon and Sorlin, Edition Frontieres) p 677 